\title{
Patient Experience with Treatment of Relapsed or Refractory Multiple Myeloma (RRMM): Content Validity of the Satisfaction and Experience Questionnaire (SEQ)-MM
}

\section{Anne Skalicky ( $\square$ anne.skalicky@evidera.com )}

Evidera Inc https://orcid.org/0000-0001-9865-3824

\section{Emre Yucel}

Amgen Inc

\section{Olabimpe Ruth Eseyin}

Evidera Inc

Aylin Yucel

Amgen Inc

\section{Sumeet Panjabi}

Janssen Pharmaceuticals Inc.

\section{Research}

Keywords: patient, treatment, satisfaction, experience, refractory/relapsed multiple myeloma, patientreported outcome measure, instrument development, content validation

Posted Date: May 5th, 2021

DOl: https://doi.org/10.21203/rs.3.rs-459634/v1

License: (c) (i) This work is licensed under a Creative Commons Attribution 4.0 International License. Read Full License 


\section{Abstract}

Background: Patients with multiple myeloma (MM) generally progress to being relapse/refractory (RR), having received several lines of treatment with various dosing frequencies and administration modalities, which impact patient satisfaction and experience. While there is still no cure, patients live longer but with potential burden attributable to treatment. A fit-for-purpose patient-reported outcome measure (PROM) assessing patient satisfaction and experience is missing for RRMM.

Methods: We developed the Satisfaction and Experience Questionnaire (SEQ) for MM to measure patient satisfaction and experience, based on concepts identified from a literature review and nurse/patient perceptions about patient experiences. Online focus groups were conducted with oncology nurses who treat patients with MM to provide expert opinion, followed by concept elicitation and cognitive patient interviews. A preliminary SEQ-MM measure was first drafted based on desk research. Patient feedback from the cognitive interviews resulted in revisions to the preliminary SEQ-MM's instructions, item wording, and response options between rounds. Content analysis was conducted to assess concept frequency mentioned during the concept elicitation focus group, and an item tracking table documented the SEQ$\mathrm{MM}$ revisions based on cognitive interviews.

Results: Three oncology nurses and 10 patients with RRMM participated in the focus groups. Oncology nurses reported treating 17 patients with MM per week, of which 33\% were patients with RRMM. Study patients were primarily male (70\%), with mean age of 56 (median range: 47 to 71 ). The most commonly reported satisfaction and experience concepts were related to: satisfaction with treatment (50\%), experience with treatment (50\%), relationship with healthcare practitioners (50\%), time burden $(50 \%)$, side effects (40\%), and avoiding relapse (40\%). The SEQ-MM was revised to include 15 items capturing: treatment burden (five items), time burden (five items), travel burden (two items), treatment compliance (one item), general satisfaction (one item), and an overall satisfaction item (one item).

Conclusions: The SEQ-MM v1.0 evaluates the important facets of satisfaction and experience in patients with RRMM receiving treatment. This tool can be used to assess facets of treatment in MM that might complement health-related quality of life data Additional work is needed to examine item performance, scoring, and psychometric properties.

\section{Background}

Multiple myeloma ( $\mathrm{MM}$ ) is an incurable, heterogenous, malignant plasma cell disorder. Most MM cases experience patterns of regression and remission, followed by relapse. In the past few decades, there have been advancements and increases in MM treatment options-specifically, providing treatment options for patients with relapse or refractory MM (RRMM) [1, 2]. The availability of multiple treatment options enables personalized treatment, especially for patients with RRMM aiming to eliminate or reduce the considerable unmet need (since the five-year survival rates are approximately 50\%) [3]. Although patients 
can find a variety of different treatments prolonging their survival, there remains a lack of measurement of patient satisfaction and experience.

Patient satisfaction and experience with a medication or medical device has been of increasing interest over the past two decades [4,5], particularly with more offerings from a wide spectrum of RRMM treatments that could potentially better fit patient lifestyles, reduce travel burden due to improved scheduling frequency, and provide safer and more efficacious dosing regimens [6]. The FDA's $21^{\text {st }}$ Century Cures Act and plan for Patient Focused Drug Development includes steps to gathering patient perspectives on burden of treatment [7-9].

Assessing current medication satisfaction gives useful insights into the patient perspective, and may aid in differentiation among alternative treatments [10]. Patient satisfaction with treatment is defined as the individual's rating of important attributes regarding the process and outcomes of their current treatment, and focuses on the interaction of expectations, preferences, and satisfaction with medical treatment [1012]. Enhancing patient satisfaction and experience with treatment modalities such as duration and dosing can improve their health outcomes and quality of life (QoL). Given the evolving changes to cancer treatments and their innovative delivery methods, understanding patients' experience and gauging their satisfaction with their cancer care is important-but difficult-to assess with existing measures which were not MM-specific and did not capture issues related to treatment modalities such as infusion, duration of administration, and perceptions around efficacy and tolerability in MM. However, there remains a lack of evidence-based data assessing the impact of these therapies on health related QoL (HRQoL) as it pertains to patient satisfaction and experience. Therefore, this knowledge gap must be addressed to better understand treatment burden within this population.

Recent studies suggest that productivity loss, time, caregiver burden, symptoms, direct costs, and treatment costs could be major drivers of patient satisfaction and experience, largely due to treatment modalities (oral vs. injectable regimen) $[13,14]$. Appropriate assessment tools to assess factors influencing patient satisfaction and experience could be invaluable in clinical decision-making as it pertains to patient adherence and could influence better patient outcomes. The implementation of treatment satisfaction assessment tools can identify issues related to treatment dissatisfaction in the complex landscape of RRMM treatments-and as a result, lead to improvements in the treatment experience of patients with RRMM [13].

Health-related quality of life (HRQoL) measures alone may not capture all relevant information about the patients' treatment experience and the treatment-related concepts they deem important, such as treatment satisfaction and convenience $[6,15,16]$. Therefore, a validated patient-reported outcome (PRO) measure for evaluating patient satisfaction and convenience is needed, especially in the current environment enwith multiple treatment options and modalities in RRMM.

While medication satisfaction may be broadly applicable, the current study focused specifically on cancer care for patients receiving RRMM treatments; in parallel, patients from other cancer types on prophylactic 
treatment for febrile neutropenia were also a focus, but are reported separately [17]. The intent of this study was to develop a tool to support decision-making related to patient satisfaction and experience; and understand what additional patient information may aid in differentiation among alternative RRMM treatments.

\section{Methods}

\section{Overview}

The SEQ-MM was developed during three stages (Figure 1). During Stage 1, a targeted literature review (TLR) was conducted to examine existing PRO measures related to patient experience and treatment satisfaction. Theoretical models of treatment satisfaction were also reviewed $[5,10,18,19]$, core concepts of interest (COls) were identified, and a preliminary conceptual framework (CF) was drafted. The $\mathrm{CF}$ included the COls guiding the development of a new measure called the Satisfaction and Experience Questionnaire-MM (SEQ-MM).

Following the drafting of the preliminary SEQ-MM v0.1, a cross-sectional observational qualitative study was conducted in two steps: 1) focus groups with oncology nurses to explore patient satisfaction and experience; and 2) focus groups and interviews with RRMM patients to first understand patient satisfaction and experience, followed by a group cognitive interview review of the draft SEQ-MM v0.1 and v0.2 to assess patient comprehension and feedback.

\section{Stage 1: Targeted Literature Review of Existing Treatment Satisfaction Measures}

During Stage 1, a TLR was conducted in two steps: 1) identify published literature reporting PRO measures that assess patient satisfaction and experience in oncology; 2) review PRO measures of patient satisfaction and experience in other indication areas beyond oncology. The search was conducted in September 2017 utilizing PubMed/MEDLINE, Embase, Ovid, and Google Scholar databases focused on English-language articles, involving only human subjects and publications between 2007 to 2017 . The key terms used were "Patient satisfaction", "Patient experience", "treatment satisfaction", "treatment experience" and "convenience"." Further information about the literature search terms can be found in the additional files (Additional file 1).

Eligible publications focused on patient-reported satisfaction and experience (including convenience) domains among patients with RRMM. Publications were ineligible if they involved pediatric patients, were not reported in English, had inaccessible full texts, did not report the use of validated PROs (or PROs were not validated among patients with cancer or hematologic malignancies), used proxy-reported outcomes, or measured other types of satisfaction (e.g., sexual satisfaction, survivor's satisfaction with healthcare delivery and accessibility, satisfaction with educational intervention, satisfaction on surgical procedure, 
etc.). Eligible articles were assessed for: 1) whether a treatment experience, convenience or satisfaction PRO was reported; 2) the PRO was relevant to the oncology population; and 3) methodological rigor and quality.

In parallel, an additional search was conducted using the same search terms on Patient-Reported Outcomes and Quality of Life Instruments Database (PROQOLID) and Functional Assessment of Chronic Illness Therapy (FACIT), which are comprehensive online databases of clinical outcome assessments (COAs) to identify generic and disease-specific PRO measures. PROs that assessed satisfaction and experience domains were eligible to be included in the search. In addition, existing health models of behavior change related to medication and treatment satisfaction and experience were reviewed. This further informed the COls to be included in the CF for patient satisfaction and experience related to treatments in RRMM.

\section{Assessment of Existing PRO Measures}

Following the literature review, information from each article was extracted, and each instrument was assessed whether it was suitable for use with patients with RRMM, captured COIs, and was relevant for the context of use. An instrument was ineligible if it did not assess the COls (patient experience and satisfaction domains such as convenience of dosing schedule, and mode of medication administration), was not validated in any population, or was not validated in patients with MM. Please review Results section that reports on brief gap analysis results of existing PRO measures.

\section{Item Development}

COls were identified based on the assessment of the identified PROs, literature review results, and review of existing health models $[5,10,18,19]$; and a pool of potential items was developed to capture patient experience and satisfaction domains; tolerability, convenience of dosing schedule, travel, treatment, bothersomeness of treatment, and satisfaction with treatment. A recall period of "most recent MM treatment" and five-point verbal rating scales (VRS) was developed. The draft items were crafted based on generally-accepted procedures outlined by Streiner and Norman [20], the International Society for Pharmacoeconomics and Outcomes Research (ISPOR) Best Practices, and the Food and Drug Administration (FDA) PRO Guidance [21-23] (Additional file 2). A Flesch-Kincaid readability assessment was conducted on the instructions and items, and revisions were made.

\section{Stage 2: Focus Group with Registered Oncology Nurses}

Registered nurses (RNs) with five or more years of oncology treatment experience in the United States (US) were recruited through a medical provider panel (www.nielsen.com) to take part in a single, 60minute, online concept elicitation focus group platform (www.focusvision.com). Participants were 
encouraged to use the video camera feature for easy identification and better interaction. A semistructured, standardized discussion guide was used to obtain RNs' assessments about patient satisfaction and experience with chemotherapy, targeted therapies, prophylactic therapies, and mode of medication administration (Additional file 3). The RNs also answered several closed- and open-ended questions, using FocusVision's online polling software, centered on patient satisfaction and experience, and tolerability based on their clinical practice experience.

$\mathrm{RN}$ input into patient satisfaction and experience with oncology treatments provided insight into the relevance and comprehensiveness of the concepts, and informed any necessary modifications to the preliminary SEQ-MM v0.1. The focus group was audio recorded (with permission) and subsequently transcribed. RNs were compensated $\$ 260$ for one hour of their time.

\section{Stage 3: Focus Groups and Interviews with RRMM Patients}

Adult patients who were US resident with a diagnosis of RRMM within the past 12 months and on current or recent (within 12 months of self-reported diagnosis date) RRMM treatment were eligible to participate in this study. Participants were required to provide physician verification of their RRMM diagnosis and treatment. These patients participated in hybrid concept elicitation and cognitive interview discussions via an online platform.

\section{Ethics and Recruitment}

This study was approved by the Quorum Review Institutional Review Board (protocol number 33649). Identification, screening, recruitment, and tracking of the patients with RRMM were managed by a thirdparty recruitment vendor, Nielsen. To participate in the vendor's panel, patients were required to submit a physician's verification of cancer diagnosis and chemotherapy medication. Eligible participants were scheduled and invited to join the discussion by the focus group vendor. Focus group participants were sent an invitation to join an online platform utilizing webcams; these interactions were audio recorded. The focus group vendor also sent all study documents to the participants, who were encouraged to use a webcam for better interaction.

\section{Discussion Guide}

A semi-structured, standardized discussion guide was used in the patient focus groups (Additional file 4). The focus group discussion guide consisted of two sections: 1) open-ended concept elicitation questions to understand patient experience with RRMM treatment and satisfaction; and 2) a cognitive interview to assess patient impressions of the relevance, comprehension, and specific feedback on the instructions, recall period, items, and response options of the SEQ-MM. Before the initiation of the cognitive interview discussion, focus group participants completed the SEQ-MM. 
A Microsoft PowerPoint presentation based on the patient semi-structured discussion guide was developed for use during the online focus groups and telephone interviews. The PowerPoint presentation allowed for discussion of treatment satisfaction and experience topics. The presentation also allowed for a review of the SEQ-MM v1.0 items and response options, alongside alternative items and response options arising from previous focus groups or interviews for patient review and consideration.

\section{Other Questionnaires}

After the patient focus group discussions, the following forms were completed by each participant: 1) the SEQ-MM Item Relevance Questionnaire, which obtained the relevance ratings for each item response options rated on a five-point VRS (not important, slightly important, moderately important, very important, extremely important); 2) a sociodemographic form, which included questions on age, race, employment, and education; 3 ) a medical history form, which included questions on disease and treatment history; and 4) a treatment characteristics questionnaire, which ascertained a general rating of treatment satisfaction, the time burden, and indirect costs of traveling to receive treatment. At the end of the focus group, selfreported demographic data was collected from participants, and the focus group vendor disbursed compensation of $\$ 175$ to study participants.

In the cognitive interview portion of the focus group, patients reviewed the instructions, item content, recall period, and response options. The item intent, meaning, and relevance was assessed for the SEQMM v0.2 items. All transcripts were developed from the audio files.

\section{Data Analysis}

Descriptive statistics (e.g., n, mean, standard deviation [SD], and/or frequency) for quantitative sociodemographic and medical history data were calculated and summarized in results tables to characterize the sample. SAS v9.4 was used for the analyses of the descriptive statistics [24].

Separate preliminary coding frameworks were developed for Stages 2 and 3 concept elicitation discussions related to the meaning of treatment experience and satisfaction. The coding frameworks were imported into ATLAS.ti v8 software to code transcripts [25]. Structured counts were taken during each focus group for each treatment experience and satisfaction concept mentioned. The concepts arising from the patient concept elicitation discussion were documented and evaluated for saturation. Saturation was defined as the point at which no substantially new themes, concept descriptions, or terms were introduced as additional discussions are conducted $[22,23,26]$.

To document saturation, the first mention of each concept was examined, and concepts were evaluated to see which were mentioned early in the concept elicitation interview (or if they arose in later discussion). Saturation was documented using a saturation grid, and displayed the first instance of emerging 
concepts in interviews/focus groups. A thematic analysis was conducted for the concept elicitation section of the discussion.

For the Stage 3 cognitive interviews, an item tracking matrix was utilized to track feedback and recommendations for changes to each item/question. Also, a coding framework for the cognitive interviews was developed. ATLAS.ti was used to document specific discussions related to the SEQ-MM and suggestions for changes, resulting in the final SEQ-MM v1.0. A content analysis approach was used to analyze and interpret the coded cognitive interview data.

\section{Results}

\section{Targeted Literature Review}

A schematic diagram reporting the results of the TLR is outlined in Figure 2. A total of 972 published abstracts on oncology treatment include PROs screened for patient experience and satisfaction concepts of patient expectation, convenience, satisfaction with medication, and adherence and tolerability to therapy. After removing duplicates, publications with only abstracts available, reviews, cases studies, or clinical guidance articles, 406 full-text articles were reviewed and assessed for eligibility. Of these, only 23 articles were deemed eligible, as they included oncology trials assessing medication interventions, PROs measureing treatment or medication convenience, tolerability, or satisfaction.

Following the literature review, a brief gap analysis was conducted on the 28 satisfaction-related questionnaires identified from the literature and key informationInstrument concepts were reviewed and mapped to concepts of interest and the specific context of use in cancer trials: convenience of medication dosing schedule, medication satisfaction, and satisfaction with mode of treatment administration. Of these 28 measures, only four were specifically related to cancer/chronic illnesses, and encompassed patient satisfaction with oncology treatment: Cancer Therapy Satisfaction Questionnaire (CTSQ) [27], Treatment Satisfaction Questionnaire for Medication (TSQM) [28], FACIT-Treatment Satisfaction-General (FACIT-TS-G), and Treatment Satisfaction with Medicines Questionnaire (SATMEDQ) [29].

Based on the review of the four identified PROs with potential concepts of interest, none were deemed suitable to assess the treatment and medication experience and satisfaction of patients with RRMM. Instead these tools included multiple domains, including satisfaction with healthcare procedures, quality metrics, information perception by patients, healthcare provider (HCP) appraisal, and provider ability to provide adequate care. Additionally, existing measures did not look at the indirect difference between once-weekly versus twice-weekly administration of an infusion-administered oncology treatment. Infusion-administered oncology treatments require patients to come back to the clinic, and would require customized step-up dose escalation, which may be forgotten or omitted.

Based on this finding, the study team decided to create a new instrument specifically for use with RRMM therapies [17] in order to more accurately capture experience, convenience, and satisfaction with the 


\section{Development of Preliminary Conceptual Framework}

An item pool of relevant items from the four identified PROs was created for potential adaptation and inclusion in the new measure. Items were revised to capture treatment experience, satisfaction, and convenience; and a preliminary CF was developed to include the RRMM treatment COls of patient experience. Patients' experience and satisfaction with treatment efficacy and safety were posited to be directly related to the experience of improving or worsening symptoms, side effects, patient adherence to treatment, and the overall burden on the patient imposed by factors such as the convenience of schedule, duration, timing of dosing, and treatment cost.

\section{Results from Nurse Focus Group}

Five RNs were recruited to participate in an online focus group. Two RNs were unable to participate at the last minute. The three participating RNs reported treating an average of 22 patients with MM per week, of which $33 \%$ were patients with RRMM. Oncology nurses described patients' satisfaction and tolerability with RRMM treatment based on their clinical practice experience. Primarily, nurses reported that patients with RRMM derived satisfaction from experiencing a treatment response or indication that the treatment was working, and from good communication with their cancer care team. The nurses stated that patients were willing to tolerate treatment side effects if they saw a treatment response or if their QoL was not significantly impacted.

\section{Draft Preliminary SEQ-MM v0.1 Tool}

Based on the additional information from the RN focus groups, the SEQ-MM v0.1 was finalized before patient testing. Fourteen items included the following domains: convenience $(n=3)$, interference $(n=5)$, satisfaction $(n=1)$, tolerability $(n=1)$, preference $(n=1)$, experience $(n=2)$, and confidence $(n=1)$. Readability of health-related measures is a core component of comprehensibility [20, 30, 31]. Using the FleschKincaid Grade Level (FKGL) test, the grade level of the SEQ-MM v0.1 was evaluated to grade 8.4.

\section{Concept Elicitation Results}

Three rounds of hybrid concept elicitation and cognitive interview focus groups were conducted to support attainment of concept saturation.

\section{Patient Characteristics}


The detailed demographics of the patient sample are reported in Table 1a. Ten RRMM study participants participated in three focus groups and one individual interview. Three rounds of focus group discussions and one interview were conducted. Five participants were in each round (Round 1: two focus groups; Round 2: one focus group and one interview). Participants were primarily male (70\%), with mean ages of $56 \pm 8$ years.

Table 1a

Patient Sociodemographic Characteristics 


\begin{tabular}{|c|c|}
\hline Characteristics & $\begin{array}{l}\text { Total } \\
(\mathrm{N}=10)\end{array}$ \\
\hline \multicolumn{2}{|l|}{ Age } \\
\hline Mean (SD), Median [Range] & $56(8), 54[47-71]$ \\
\hline \multicolumn{2}{|l|}{ Gender, (\%) } \\
\hline Male & $7(70 \%)$ \\
\hline \multicolumn{2}{|l|}{ Ethnicity, n (\%) } \\
\hline Hispanic or Latino & $1(10 \%)$ \\
\hline Not Hispanic or Latino & $9(90 \%)$ \\
\hline \multicolumn{2}{|l|}{ Racial Background, n (\%) } \\
\hline Black or African American & $1(10 \%)$ \\
\hline White & $9(90 \%)$ \\
\hline \multicolumn{2}{|l|}{ Employment Status, $\mathrm{n}(\%)^{\bigotimes}$} \\
\hline Employed, full-time & $5(50 \%)$ \\
\hline Employed, part-time & $1(10 \%)$ \\
\hline Unemployed & $2(20 \%)$ \\
\hline Retired & $1(10 \%)$ \\
\hline Disabled & $2(20 \%)$ \\
\hline \multicolumn{2}{|l|}{ Highest Level of Education, n (\%) } \\
\hline Some college & $1(10 \%)$ \\
\hline College degree & $7(70 \%)$ \\
\hline Post graduate degree & $2(20 \%)$ \\
\hline \multicolumn{2}{|l|}{ Insurance Status, n (\%) } \\
\hline Insured, minimal out-of-pocket costs & $7(70 \%)$ \\
\hline Insured, significant out-of-pocket costs & $3(30 \%)$ \\
\hline${ }^{\mathbb{R}}$ Responses not mutually exclusive & \\
\hline
\end{tabular}

Half of participants reported receiving their cancer diagnosis over three years prior to screening $(n=5$, $50 \%)$. Most RRMM treatments were received subcutaneously ( $n=7,70 \%)$, and the majority reported receiving lenalidomide $(n=8,80 \%)$, dexamethasone $(n=7,70 \%)$, or bortezomib $(n=6,60 \%)$. Most 
participants were insured and paid minimal out-of-pocket costs $(n=7,70 \%)$. Detailed medical information is reported in Table $1 \mathrm{~b}$.

\section{Table 1b}

Medical History 
How many years ago did you receive the multiple myeloma diagnosis?

\section{1 - 2 years}

2 - 3 years

$3+$ years

$5(50 \%)$

Missing

Has your cancer spread to another region or organ? Yes

Are you currently receiving treatment? Yes

Can you recall the name of the treatment you are taking/you received? ${ }^{\rrbracket}$

Kyprolis ${ }^{\circledR}$ (carfilzomib)

Velcade ${ }^{\circledR}$ (bortezomib)

Ninlaro® (ixazomib)

Revlimid $\circledast$ (lenalidomide)

$8(80 \%)$

Pomalyst $\circledast$ (pomalidomide)

Synovir ${ }^{\circledR}$, Thalomid $\circledast$ (thalidomide)

Empliciti ${ }^{\circledR}$ (elotuzumab)

Cytoxan ${ }^{\circledR}$, Neosar ${ }^{\circledR}$ (cyclophosphamide)

Prednisone

Dexamethasone

$7(70 \%)$

Others (Please specify)*

What date was your last RRMM treatment?

$<6$ months

6 months - 1 year

1 - 2 years

Missing

How is/was your RRMM treatment administered?

Subcutaneous (SC)

Intravenous (IV) 
Have you been diagnosed by a medical doctor with any other serious health conditions besides your cancer?

No other health condition

Kidney disease

Other serious health condition $†$

In general, how would you say your health was within the past week?

Very good

Good

Fair

${ }^{\mathbb{R}}$ Responses not mutually exclusive

* Other treatment includes: Daralex, Daratumumab, Veneloclax

† Other serious condition include: High Blood Pressure

\section{Experiences with Treatment}

Generally, participants relayed positive experiences with treatment-knowing their treatment is working $(n=8,80 \%)$, not experiencing side effects $(n=7,70 \%)$, having a good relationship with their HCP $(n=6$, $60 \%)$, and having a good quality of life $(n=3,30 \%)$. Individual participants $(10 \%)$ described positive treatment experiences as receiving treatment close to home and from prominent HCPs or medical facilities (Table 2).

Table 2

Experience with MM Treatment 


$\begin{aligned} & \text { Concepts related } \\ & \text { to treatment } \\ & \text { experience }\end{aligned}$
n*

$\begin{array}{lll}\text { Medication } & 8 & 80 \%\end{array}$
working/treatment response

"Well, I guess the overarching most positive aspect of the treatment experience is so far it seems to be effective and seems to be keeping everything stable at this point."

- Male, 58 years

Side effects (pain, $7 \quad 70 \% \quad$ "My pain is subsiding, ... [my HCP is] able to help me control them... fatigue, diarrhea, be able to control those side effects so that I can go about my nausea, hair falling out) day." - Female, 53 years

$\begin{array}{ll}\begin{array}{l}\text { Relationship with } \quad 6 \quad 60 \% \\ \mathrm{HCP}\end{array} & \begin{array}{l}\text { "I would also add that I feel the communication with my physician } \\ \text { is a huge positive to my experience. We dialogue about options. } \\ \text { I'm never in a position where I'm told what to do as much as we } \\ \text { consult about how treatment should progress and what options } \\ \text { are available before we make any decisions." } \\ \text { - Female, 55 years }\end{array}\end{array}$

Treatment time $5 \quad 50 \% \quad$ "Other than having to come in often on some of the twice a week burden (including sometimes is an inconvenience. It's down to just once a month travel time) now so that's a good thing. The number of times you have to go in for treatment is a concern." - Male, 71 years

Quality of life $\quad 3 \quad 30 \% \quad$ "Most important factors, I mean in perfect environment it would be something that would respond well to the myeloma. It would be something that could either be done at home or not as frequent. Maybe biweekly or monthly would be optimal and something that would allow us to have a quality of life so that we can be around others, we can be with our family members. We can really just live our life while we're going through the treatment." - Female, 47 years

$\begin{aligned} & \text { Disease } \\ & \text { progression }\end{aligned} \quad 3 \quad 30 \% \quad \begin{aligned} & \text { "Well, after my stem cell transplant became ineffective and I went } \\ & \text { into relapse it was then a question as to what would be the course } \\ & \text { of action to take next. Obviously having something to take was } \\ & \text { important to me to get back into remission or as close to } \\ & \text { remission as possible." - Male, } 71 \text { years }\end{aligned}$

Convenient location of treatment site

Prominent hospital
$1 \quad 10 \% \quad$ "...my hospital is close to my office so I can keep my schedule when I go in for treatment. They're very flexible, ... so I would-I would say for the most part... it's been positive."

- Male, 48 years

$1 \quad 10 \%$
"I love my doctor...the nurses, the staff generally care. It's a very prominent hospital... their teams are some of the best -in the country and I-I trust them with patient welfare." - Male, 48 years

\section{* Number and percent of participants who endorse a concept}

Participants commented on how a few RRMM treatment experiences can have both positive and negative aspects. Participants reported negative experiences from not seeing a treatment response $(n=8,80 \%)$, 
experiencing side effects $(n=7,70 \%)$, or experiencing disease progression $(n=3,30 \%)$. Participants also viewed the time burden of treatment, including travel time $(n=5,50 \%)$, as a negative experience.

\section{Satisfaction with Treatment}

When asked about treatment satisfaction, participants defined this as whether the RRMM treatment is a repeated positive or negative experience (Table 3). Reasons for participants' RRMM treatment satisfaction included the medication working/treatment response $(n=9,90 \%)$, relationship with HCP ( $n=4$, $40 \%)$, disease remission ( $n=2,20 \%)$, maintaining quality of life $(n=2,20 \%)$, and getting treatment at a prominent hospital $(n=1,10 \%)$. Reasons for dissatisfaction with RRMM treatment included disease progression $(n=5,50 \%)$, side effects $(n=4,40 \%)$, treatment time burden $(n=3,30 \%)$, and treatment cost $(n=1,10 \%)$.

Table 3

Satisfaction/Dissatisfaction with MM Treatment 


$\begin{aligned} & \text { Concepts related to } \\ & \text { meaning of } \\ & \text { satisfaction }\end{aligned}$

Satisfaction with

Treatment

Medication $9990 \% \quad$ "Well, I mean the ultimate satisfaction would be the effectiveness Working/Treatment of the treatment, it's working, is it working, is it not working? So Response far, my treatment seems to be working pretty well so therefore that is the ultimate satisfaction. That is why l'm doing this. That is why l'm disrupting my life and keeping this going on." - Male, 58 years

$\begin{aligned} & \text { Relationship with } \\ & \text { HCP }\end{aligned} \quad 4 \quad 40 \% \quad \begin{aligned} & \text { "I'm satisfied knowing that I have one of the top doctors in the } \\ & \text { country treating me." }\end{aligned}$
Male, 57 years

Disease Remission $2 \quad 20 \% \quad$ "Obviously having something to take was important to me to get back into remission or as close to remission as possible." - Male, 71 years

Maintaining Quality of Life

$220 \%$

Prominent Hospital $1 \quad 10 \%$

Prominent Hospital $1 \quad 10 \%$

"I think it's... the ability to conduct myself at the highest level on a regular basis, so quality of life. Can I get up this morning and do what I need to do, eat fairly routine normal food, sleep well." Female, 55 years

"Oftentimes the negative has been the frequency of my treatment. My specialist is about 140 miles away and so when I'm there twice a week it's a lot. Sometimes we are there for treatment twice a week, sometimes we're there for simple blood work and other treatments. When I'm hospitalized I go to that hospital as well which is over by my specialist. Everything for me is out of town but that's by choice. I mean that's where I prefer to get my treatment." - Female, 47 years

Dissatisfaction with Treatment

\begin{tabular}{|c|c|c|c|}
\hline $\begin{array}{l}\text { Disease } \\
\text { Progression }\end{array}$ & 5 & $50 \%$ & $\begin{array}{l}\text { "Well for me personally, at this point, um, I'm not satisfied } \\
\text { because I have not hit remission at any point... the best I've } \\
\text { gotten is...very good response, um, so it's been very, very tough for } \\
\text { me. I would say I'm dissatisfied... with my experience because the } \\
\text { disease is just so-it seems to be so strong... every therapy that } \\
\text { we've tried...I've relapsed." - Female, } 53 \text { years }\end{array}$ \\
\hline Side Effects & 4 & $40 \%$ & $\begin{array}{l}\text { "You're dissatisfied if... you get some side effects that are } \\
\text { unbearable or are such that even with the satisfactory number } \\
\text { situation that you feel like it's not tolerable." - Male, } 71 \text { years }\end{array}$ \\
\hline $\begin{array}{l}\text { Treatment Time } \\
\text { Burden }\end{array}$ & 3 & $30 \%$ & $\begin{array}{l}\text { "The treatments I receive are four or five hours at a time. That's a } \\
\text { full day shot. If you're doing that twice a week it'd be very hard to } \\
\text { maintain a job." - Male, } 71 \text { years }\end{array}$ \\
\hline
\end{tabular}




\begin{tabular}{|c|c|c|c|}
\hline $\begin{array}{l}\text { Concepts related to } \\
\text { meaning of } \\
\text { satisfaction }\end{array}$ & $\mathrm{n}^{*}$ & $\%$ & Patient quote \\
\hline Treatment Cost & 1 & $10 \%$ & $\begin{array}{l}\text { "One thing to me that I would again put under the dissatisfying } \\
\text { category, would be sometimes the cost. Lord it's expensive to } \\
\text { have myeloma. I think we all know that. I have pretty good } \\
\text { insurance but there I still a lot of out of pocket cost and that's a } \\
\text { little dissatisfying certainly, but it's part of the treatment." } \\
\text { - Male, } 58 \text { years }\end{array}$ \\
\hline
\end{tabular}

\section{Cognitive Interview Results}

The SEQ-MM v0.1 and v0.2 were reviewed in two rounds. Across the rounds, it took participants approximately five minutes to complete the questionnaire. Patient feedback from the cognitive interview portion of the focus group resulted in revisions to the measure.

Participants described the questionnaire and instructions as "simple," "easy to understand,"

"straightforward," and "decent." Participants spontaneously commented that using a five-point VRS works well for the questionnaire. Based on patient feedback, the response options of some items were also revised, as about $70 \%$ of patients preferred the five-point VRS over the three-point scale or 11-point numeric rating scale (NRS); they indicated their preference for a consistent scale throughout the questionnaire. All participants were clear on the item recall periods and understood the phrase "most recent." After the second round, the SEQ-MM v0.2 was revised to include 15 items capturing the item "time spent receiving treatment." Additional file 5 provides the item history, summarizing revisions made to the newly-developed SEQ-MM v1.0.

\section{Revised CF}

The CF for the SEQ-MM v1.0, linking the individual items to the hypothesized domains, was revised based on focus group feedback (Figure 3). The final SEQ-MM v1.0 includes 15 items (Additional file 6). Twelve items capture treatment experience: treatment burden (five items), time burden (five items), and travel burden (two items). Additional single items include: treatment compliance (one item), general health (one item), and an overall satisfaction item (one item).

\section{Other Questionnaire Results}

\section{Satisfaction with Care}


The majority of participants $(90 \%)$ rated their cancer care team as seven or higher on an 11-point NRS (worst to best cancer care team). Most participants (70\%) felt they received benefit from the treatment, with a few side effects $(60 \%)$, many side effects $(20 \%)$, or no side effects $(20 \%)$. All participants stated that they were able to tolerate the treatment despite the side effects. All participants reported that their cancer care team clearly explained their treatment in person $(90 \%)$, or in addition, provided written materials (30\%).

\section{Time Burden}

The majority (70\%) reported that office/clinic visits were scheduled at times that were convenient for them; $30 \%$ said that times were sometimes convenient for them. On average, patients spent 174 minutes (126 SD; range 66 to 360 ) in the clinic to receive treatment. Participants reported taking about 48 minutes (66 SD; range 18 to 240 ) to travel one way to receive treatment, with $80 \%$ of patients traveling one to three times per month. Most participants (80\%) reported driving themselves to treatment, and $60 \%$ were accompanied by another person.

\section{Indirect Costs}

Participants reported paying for gas (80\%), parking (30\%), or taxi fare to the clinic (30\%). One participant $(10 \%)$ reported needing to find temporary lodging to receive treatment. On average, patients paid $\$ 16.50$ (\$15.50 SD; range \$1 to \$50) for gas, \$10 (\$5.00 SD; range \$5 to \$15) for taxi fare, \$16.50 (\$19.10 SD; range $\$ 3$ to $\$ 30)$ for parking, and $\$ 200$ for lodging. Four participants (40\%) reported that insurance covered all costs, and six participants (60\%) reported out-of-pocket treatment costs ranging from $\$ 50$ to $\$ 6,000$ with a mean of $\$ 1,764.20(\$ 2,351$ SD).

\section{Discussion}

The SEQ-MM v1.0 was developed iteratively, including FDA and ISPOR best practice methods $[9,22,23]$. The concepts included in the SEQ-MM were supported by feedback from oncology nurses and patients with RRMM regarding treatment experience. The focus group discussion with oncology nurses supported RRMM patient feedback that indicates that patients derived satisfaction from experiencing a treatment response, and from good communication with their cancer care team. Nurses stated that patients were willing to tolerate treatment side effects if they saw a treatment response, or if the time burden or their QoL was not significantly impacted. Patients described that worthwhile treatment meant there is positive treatment response, minimal side effects, and minimal time burden.

The instrument development process (including creation of questions, recall period, and response choices) followed published guidelines and procedures [21-23]. The study results demonstrate that participants with RRMM were able to understand the SEQ-MM v1.0 instructions, items, and response options. Feedback from 10 patients confirmed the content validity of the item concepts as relevant to 
their treatment experience and satisfaction. Additionally, cognitive interviews provided supportive evidence of patient satisfaction with treatment benefit; minimal side effects as important to SEQ-MM v1.0 concepts was supported by the additional questionnaire results. Participants reported lengthy oneway travel times and treatment wait times, and many were accompanied by another person predominantly at times convenient to them. Participants all described out-of-pocket indirect costs for transportation, and a majority reported significant out-of-pocket treatment costs.

\section{Limitations}

The content validity assessment was carried out with a sample of 10 adults with RRMM, which is an adequate sample size for reaching saturation for a relatively uncommon cancer. The study participants reviewing the SEQ-MM included patients with RRMM; these results are not generalizable to other tumor types and diseases. Study participants reported having received several MM treatments, with distinct modes of medication delivery.

Scheduling of focus groups sessions, punctuality, and delays can affect the onset and attendance of focus group sessions. In this study, only three of the five RNs were able to attend the session. However, online, video-enabled focus groups allowed for easy rapport-building, screen-sharing of visual aids, and participation of diverse patients that may not have participated in the in-person focus groups. The number of participants in each focus group session differed, resulting in fewer topics/questions being covered in focus groups with more participants. While individual in-person cognitive interviews may be ideally suited for detailed cognitive interview feedback, the quality of patient input can still be achieved using focus group design and using online methods. The study only included two rounds of cognitive interview discussions. A third round of cognitive interviews was not possible; however, the feedback for changes in the second round of focus groups and the individual interview was supportive of the final SEQ-MM revisions.

In the future, the SEQ-MM will need to undergo continued patient testing to assess its generalizability to newly diagnosed $\mathrm{MM}$ and other tumor types in relation to their treatment experience and treatment satisfaction. This way, it can be a useful tool in comparative effectiveness or treatment evaluation trials.

\section{Conclusion}

This study reports on the development and content validity of the patient-reported SEQ-MM v1.0, which provides insights into the patient's experience of burden of current oncology treatment, as well as satisfaction with that experience. The tool was initially developed based on concepts identified from a literature review of oncology measures, interviews with oncology nurses and later revised and tested in a sample of and RRMM patients undergoing treatment. The instrument captures the core aspects of patient cancer care experience, and related overall satisfaction with that experience among patients with RRMM and may complement data gathered from traditional HRQoL measures. 
Next steps should ensure continued validation of the PROM for its clinical relevance in the face of new $\mathrm{MM}$ treatments such as B-cell maturation antigen-targeting ones that are being developed. Translation of the SEQ-MM v1.0 is needed as well as an examination of the measure's item performance, scoring, and psychometric properties. Additionally, future revisions or adaptations to the SEQ-MM v1.0 should be assessed for content validity, clinical relevance and psychometric properties for measuring patients' perceptions of treatment experience, satisfaction, and convenience for use as a clinical trial endpoint.

\section{Abbreviations}

CF, conceptual framework; COA, clinical outcome assessments; COI, concepts of interest; CTSQ, Cancer Therapy Satisfaction Questionnaire; FACIT, Functional Assessment of Chronic Illness Therapy; FACIT-TSG, FACIT-Treatment Satisfaction-General; FDA, Food and Drug Administration; FKGL, Flesch-Kincaid Grade Level; HCP, healthcare provider; HRQoL, health-related quality of life; ISPOR, International Society for Pharmacoeconomics and Outcomes Research; MM, multiple myeloma; NRS, numeric rating scale; PRO, patient-reported outcome; PROQOLID, Patient-Reported Outcomes and Quality of Life Instruments Database; PROM, patient-reported outcome measure; QoL, quality of life; RN, registered nurses; RR, relapse/refractory; RRMM, relapsed or refractory multiple myeloma; SATMED-Q, Treatment Satisfaction with Medicines Questionnaire; SD, standard deviation; SEQ, Satisfaction and Experience Questionnaire; TLR, targeted literature review; TSQM, Treatment Satisfaction Questionnaire for Medication; US, United States; VRS, verbal rating scale

\section{Declarations}

\section{Ethics approval and consent to participate}

This study was reviewed and approved by Quorum Review Institutional Review Board (approval number 33649). All participants signed an informed consent form before any study-related focus group discussions or interview were conducted.

\section{Consent for publication}

Not applicable

\section{Availability of data and material}

Qualified researchers may request data from Amgen clinical studies. Complete details are available at http://www.amgen.com/datasharing. The SEQ-MM can be obtained for use by contacting the authors.

\section{Competing interests}


EY, AY, and SP are employees and stockholders of Amgen Inc. AS and ORE are employees of Evidera Inc.

\section{Funding}

This study was funded by Amgen Inc. The funder contributed to study design, data collection, data analysis, and data interpretation.

\section{Authors' contributions}

EY, AY, SP: Study concept and design, data analysis, data interpretation, report writing. AS, ORE: Study concept and design, data acquisition, data analysis, data interpretation, report writing. The corresponding author had full access to all data in the study, and had the final responsibility for the decision to submit for publication.

\section{Acknowledgments}

Writing support was funded by Amgen Inc. The study team would like to thank the oncology nurses and RRMM patients who participated in the focus groups or in-depth interviews. Thanks also go to Evidera research team, Jun Chen for support with data analysis, and Fritz Hamme and Dawn Ri'chard for manuscript editorial support.

\section{References}

1. Pawlyn C, Davies FE. Toward personalized treatment in multiple myeloma based on molecular characteristics. Blood. 2019 Feb 14;133(7):660-75.

2. Sonneveld P, Avet-Loiseau H, Lonial S, Usmani S, Siegel D, Anderson KC, et al. Treatment of multiple myeloma with high-risk cytogenetics: a consensus of the International Myeloma Working Group. Blood. 2016 Jun 16;127(24):2955-62.

3. Solimando AG, Da Via MC, Cicco S, Leone P, Di Lernia G, Giannico D, et al. High-Risk Multiple Myeloma: Integrated Clinical and Omics Approach Dissects the Neoplastic Clone and the Tumor Microenvironment. J Clin Med. 2019 Jul 9;8(7).

4. Salame N, Perez-Chada LM, Singh S, Callis Duffin K, Garg A, Gottlieb AB, et al. Are Your Patients Satisfied A Systematic Review of Treatment Satisfaction Measures in Psoriasis. Dermatology. 2018;234(5-6):157-65.

5. Shikiar R, Rentz AM. Satisfaction with medication: an overview of conceptual, methodologic, and regulatory issues. Value Health. 2004 Mar-Apr;7(2):204-15.

6. Moreau P, Kumar S, Boccia R, lida S, Goldschmidt H, Cocks K, et al. Convenience, satisfaction, healthrelated quality of life of once-weekly $70 \mathrm{mg} / \mathrm{m}(2)$ vs. twice-weekly $27 \mathrm{mg} / \mathrm{m}(2)$ carfilzomib 
(randomized A.R.R.O.W. study). Leukemia. 2019 Dec;33(12):2934-46.

7. 21ST Century Cures Act, H.R. 34, 114TH Cong 2016.

8. Food Drug Administration. FDA Patient-Focused Drug Development Guidance Series for Enhancing the Incorporation of the Patient's Voice in Medical Product Development and Regulatory Decision Making. 2020 [updated 2020; cited 2021 April 6]; Available from:

https://www.fda.gov/drugs/development-approval-process-drugs/fda-patient-focused-drugdevelopment-guidance-series-enhancing-incorporation-patients-voice-medical.

9. Food Drug Administration. Patient-Focused Drug Development: Collecting Comprehensive and Representative Input. 2018 [updated 2018; cited 2021 April 6]; Available from:

https://www.fda.gov/regulatory-information/search-fda-guidance-documents/patient-focused-drugdevelopment-collecting-comprehensive-and-representative-input.

10. Weaver M, Patrick DL, Markson LE, Martin D, Frederic I, Berger M. Issues in the measurement of satisfaction with treatment. Am J Manag Care. 1997 Apr;3(4):579-94.

11. Pascoe GC. Patient satisfaction in primary health care: a literature review and analysis. Eval Program Plann. 1983;6(3-4):185-210.

12. Weldring T, Smith SMS. Patient-Reported Outcomes (PROs) and Patient-Reported Outcome Measures (PROMs). Health Services Insights. 2013;6:61-8.

13. Chari A, Romanus D, DasMahapatra P, Hoole M, Lowe M, Curran C, et al. Patient-Reported Factors in Treatment Satisfaction in Patients with Relapsed/Refractory Multiple Myeloma (RRMM). Oncologist. 2019 Nov;24(11):1479-87.

14. Sparano F, Cavo M, Niscola P, Caravita T, Efficace F. Patient-reported outcomes in relapsed/refractory multiple myeloma: a systematic review. Support Care Cancer. 2018 Jul;26(7):2075-90.

15. Revicki DA. Patient assessment of treatment satisfaction: methods and practical issues. Gut. 2004 May;53 Suppl 4:iv40-4.

16. Barbosa CD, Balp MM, Kulich K, Germain N, Rofail D. A literature review to explore the link between treatment satisfaction and adherence, compliance, and persistence. Patient Prefer Adherence. 2012;6:39-48.

17. Yucel A, Skalicky A, Eseyin OR, Yucel E, Belani R, Bensink M. Development and Content Validation of the Satisfaction and Experience Questionnaire for Granulocyte Colony-Stimulating Factor (SEQ-GCSF). Submitted to JPRO (Under Review).

18. Atkinson MJ, Kumar R, Cappelleri JC, Hass SL. Hierarchical construct validity of the treatment satisfaction questionnaire for medication (TSQM version II) among outpatient pharmacy consumers. Value Health. 2005 Nov-Dec;8 Suppl 1:S9-S24.

19. Glanz K, Rimer BK, Lewis FM. Health Behavior and Health Education. Theory, Research and Practice. San Fransisco: Wiley \& Sons; 2002.

20. Streiner DL, Norman GR. Health Measurement Scales: A Practical Guide to their Development and Use. Oxford: Oxford University Press; 2008. 
21. Food Drug Administration (FDA). Guidance for industry patient-reported outcome measures: use in medical product development to support labeling claims. Fed Regist. 2009;74(235):65132-3.

22. Patrick DL, Burke LB, Gwaltney CJ, Leidy NK, Martin ML, Molsen E, et al. Content validity-establishing and reporting the evidence in newly developed patient-reported outcomes (PRO) instruments for medical product evaluation: ISPOR PRO good research practices task force report: part 1-eliciting concepts for a new PRO instrument. Value Health. 2011a Dec;14(8):967-77.

23. Patrick DL, Burke LB, Gwaltney CJ, Leidy NK, Martin ML, Molsen E, et al. Content validity-establishing and reporting the evidence in newly developed patient-reported outcomes (PRO) instruments for medical product evaluation: ISPOR PRO Good Research Practices Task Force report: part 2-assessing respondent understanding. Value Health. 2011b Dec;14(8):978-88.

24. SAS Institute Inc. SAS/GRAPH 9.4. Raleigh, NC; 1979-2018.

25. Friese S, Ringmayr TG. ATLAS.ti 8 Windows User Manual. Berlin: ATLAS.ti Scientific Software Development $\mathrm{GmbH} ; 2019$.

26. Kerr C, Nixon A, Wild D. Assessing and demonstrating data saturation in qualitative inquiry supporting patient-reported outcomes research. Expert Rev Pharmacoecon Outcomes Res. 2010 Jun;10(3):269-81.

27. Abetz L, Coombs JH, Keininger DL, Earle CC, Wade C, Bury-Maynard D, et al. Development of the cancer therapy satisfaction questionnaire: item generation and content validity testing. Value Health. 2005 Nov-Dec;8 Suppl 1:S41-53.

28. Atkinson MJ, Stewart WC, Fain JM, Stewart JA, Dhawan R, Mozaffari E, et al. A new measure of patient satisfaction with ocular hypotensive medications: the Treatment Satisfaction Survey for Intraocular Pressure (TSS-IOP). Health Qual Life Outcomes. 2003 Nov 15;1:67.

29. Ruiz MA, Pardo A, Rejas J, Soto J, Villasante F, Aranguren JL. Development and validation of the "Treatment Satisfaction with Medicines Questionnaire" (SATMED-Q). Value Health. 2008 SepOct;11(5):913-26.

30. Petkovic J, Epstein J, Buchbinder R, Welch V, Rader T, Lyddiatt A, et al. Toward Ensuring Health Equity: Readability and Cultural Equivalence of OMERACT Patient-reported Outcome Measures. J Rheumatol. 2015 Dec;42(12):2448-59.

31. Kincaid JP, Fishburne RP, Rogers RL, Chissom BS. Derivation of New Readability Formulas (Automated Readability Index, Fog Count and Flesch Reading Ease Formula) for Navy Enlisted Personnel. Millington, TN: Naval Air Station Memphis; 1975.

\section{Figures}




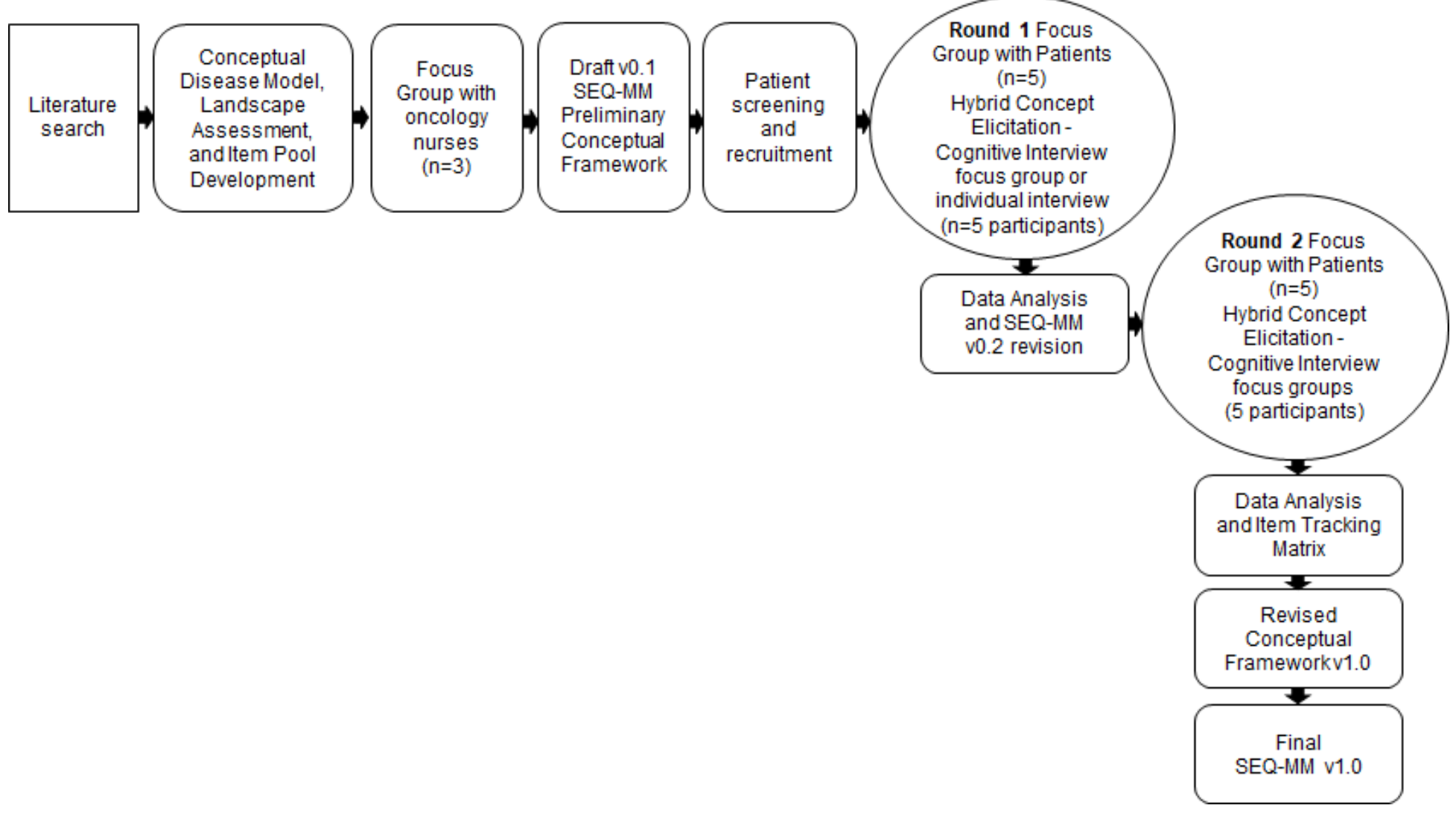

\section{Figure 1}

Study Design Schema

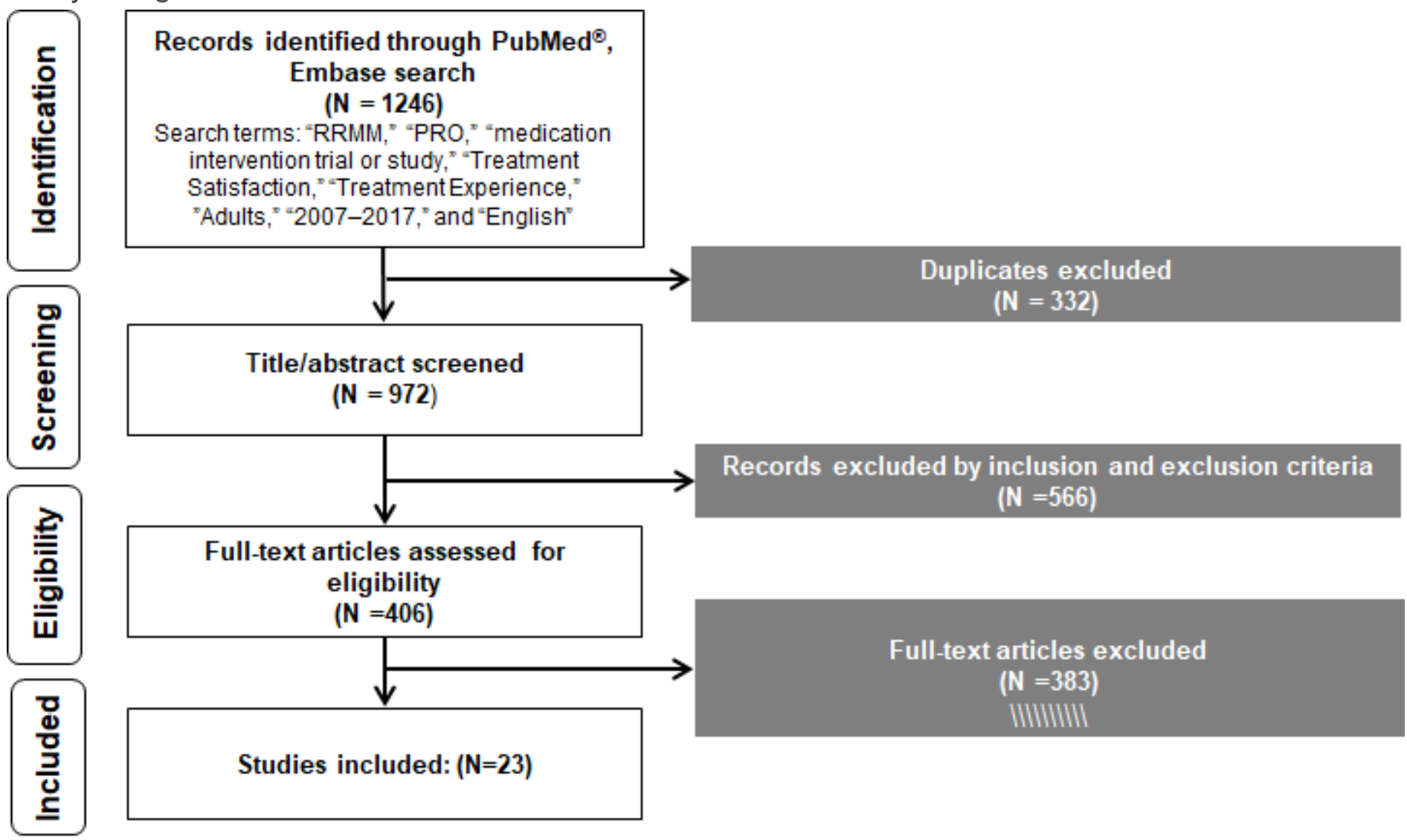




\section{Figure 2}

Flow Diagram for Targetted Literature Review

\begin{tabular}{|l|}
\hline Overall satisfaction with treatment \\
\hline Overall rating of health since start of treatment \\
\hline Overall rating of change in health condition since start of treatment \\
\hline Overall convenience with dosing schedule \\
\hline Overall recommendation of the treatment to another patient \\
\hline Overall rating of experience with treatment \\
\hline Tolerate most recent treatment \\
\hline Concern about missing most recent treatment \\
\hline Convenient to travel to receive most recent treatment \\
\hline Convenientto make travel arrangements to mostrecent treatment \\
\hline Satisfaction with time spent to receive most recent treatment \\
\hline Bothered by time it took to receive most recent treatment \\
\hline Amount of time mostrecent treatmenttook away from daily activities \\
\hline Convenientto schedule an appointmentfor mostrecent treatment \\
\hline Convenient to receive most recent treatment \\
\hline
\end{tabular}

Treatment Experience and Satisfaction

\section{Figure 3}

SEQ-MM v1.0 Conceptual Framework

\section{Supplementary Files}

This is a list of supplementary files associated with this preprint. Click to download.

- A.TargetedLiteratureSearch.docx

- B.Preliminaryconceptualframeworkv0.1.pptx

- C.RNInterviewDiscussionGuide.docx

- D.PatientInterviewDiscussionGuide.docx

- E.SEQMMltemHistory.docx

- F.SEQMMv1.0.docx 